\title{
Development of a Prognostic Nomogram for Patients with Lung Adenocarcinoma in the Stages I, II, and III Based on Immune Scores
}

\author{
Hui Xie ${ }^{1,2}$ \\ Jian-Fang Zhang ${ }^{3}$ \\ Qing $\mathrm{Li}^{2,4}$ \\ 'Department of Radiation Oncology, \\ Affiliated Hospital (Clinical College) of \\ Xiangnan University, Chenzhou, 423000, \\ Hunan Province, People's Republic of \\ China; ${ }^{2}$ Key Laboratory of Medical \\ Imaging and Artificial Intelligence of \\ Hunan Province, Chenzhou, 423000, \\ Hunan Province, People's Republic of \\ China; ${ }^{3}$ Department of Physical \\ Examination, Beihu Centers for Disease \\ Control and Prevention, Chenzhou, \\ 423000, Hunan Province, People's \\ Republic of China; ${ }^{4}$ Department of \\ Interventional Vascular Surgery, Affiliated \\ Hospital (Clinical College) of Xiangnan \\ University, Chenzhou, 423000, Hunan \\ Province, People's Republic of China
}

Correspondence: Qing Li Department of Interventional Vascular Surgery, Affiliated Hospital (Clinical College) of Xiangnan University, 25

Renmin Street, Chenzhou, 423000 ,

Hunan Province, People's Republic of China

Tel +86 19918761912

Email xnxyliqing@।63.com
Background: Immunotherapy has significantly changed the treatment prospects of nonsmall cell lung cancer (NSCLC). However, there is no report based on immune score to predict the overall survival (OS) of lung adenocarcinoma (LUAD) in the stages I, II, and III. Therefore, this study aimed to investigate the immune score and the prognosis-related factors of LUAD and construct a nomogram to predict the prognosis.

Methods: A total of 390 cases with lung adenocarcinoma in the stages I, II, and III were included in the study. The clinicopathological characteristics and immune scores of LUAD patients were downloaded from the TCGA database. Cox proportional hazards regression model was used to estimate hazard ratio (HR) and 95\% confidence interval (CI). A Nomogram was composed of the Cox model and internally validated using 1000 bootstrap. The concordance index (c-index) and the calibration curves were used to evaluate the model. The decision curve analysis (DCA) was performed to evaluate the clinical practical value of the model.

Results: According to the immune score, the patients were divided into low-, medium-, and high-score groups. This study showed that compared with patients with low and medium immune scores, only patients with high immune scores had significantly improved OS (HR and 95\% confidence interval (CI): 0.489 [0.324-0.737]). The C-index for OS prediction was 0.691 (95\% CI, 0.646-0.736). The calibration curves for nomogram-predicted probabilities of 3- and 5-year survival have good ability for the calibration and discrimination.

Conclusion: The high immune score was significantly correlated with better OS of patients with LUAD in the stages I, II, and III. Moreover, the nomogram of predicting prognosis may help assess the survival of LUAD patients.

Keywords: lung adenocarcinoma, immune scores, nomograms, prognosis

\section{Background}

Updated statistics of lung cancer in China (2019.1) showed that lung cancer had the highest incidence and mortality rate, ranking first among all types of malignant tumors in China. It was estimated that there were 787,000 new lung cancer cases and 631,000 deaths from lung cancer in China in $2015 .^{1}$ The recently released data of the global cancer burden in 2017 showed that the incidence rate of lung cancer ranked second among all types of cancer, and the mortality rate of lung cancer was the highest in the world. ${ }^{2}$

The standard treatment of lung cancer is multidisciplinary, including traditional surgery, chemotherapy and radiotherapy, which are still effective methods for the treatment of lung cancer. 
In recent years, significant progress has been made in molecular targeted therapy and immunotherapy of lung cancer. These new methods have brought major changes to the treatment of lung cancer and improved the quality of life and survival rate of patients. ${ }^{3}$ Non-small cell lung cancer (NSCLC) accounts for about $80 \%$ of all types of lung cancers, and lung adenocarcinoma (LUAD) accounts for about $40-50 \%$ of NSCLC. ${ }^{4}$ It has been reported that LUAD usually has some invasive subtypes. LUAD patients with epidermal growth factor receptor (EGFR) mutations have a response to anti-EGFR targeted therapy. ${ }^{5}$ Recently, Japanese scholars have studied the immunophenotype of LUAD patients with EGFR mutation in the tumor microenvironment (TME), where LUAD patients with EGFR mutation were treated by antiprogrammed cell death protein 1 (PD-1) antibody monotherapy, while this method was basically ineffective. ${ }^{6}$ Therefore, targeted therapy was only effective for some appropriately selected patients with LUAD. ${ }^{7}$

Considering the advantages of recognizing the immune response in solid tumors and the immunological changes during the immunotherapy of LUAD, immunotherapy has showed advantages in the treatment of lung cancer. Among them, PD-1 inhibitors that block the programmed cell death protein 1 (PD-1)/its ligand (PD-L1) pathway have long been the first-line treatment drugs for advanced non-small cell lung cancer with positive PD-L1 expression. This therapy significantly improved the prognosis of NSCLC patients and increased the 5-year survival rate of NSCLC patients to $15.5-23 \% .^{8}$ In a clinical trial, 305 patients with high expression of PD-1 were randomly enrolled, and were given pembrolizumab at a fixed dose of $200 \mathrm{mg}$ every 3 weeks for 35 cycles or until disease progression, which were compared with chemotherapy drugs. The progression-free survival rate and overall response rate of chemotherapy drugs were significantly improved. ${ }^{9}$ Recent studies have shown that PD-1 is highly expressed in non-small cell lung cancer. ${ }^{10}$

Therefore, it is of great significance to understand the relationship between the immune system and prognosis, which can help us make more effective use of immunooncology, promote the development of this discipline, and improve the efficacy of treating tumors. The Li's research team was the first in China to propose a model based on immune score to predict the survival and the effects of adjuvant chemotherapy in patients with gastric cancer after surgery, and predict whether patients with stage II and stage III gastric cancer can benefit from adjuvant chemotherapy. ${ }^{11}$ In addition, Wang et al studied the application of immune score in the prognosis of breast cancer. ${ }^{12}$ However, studies on the prognosis of LUAD based on immune scores have not been reported yet. Therefore, this study aimed to evaluate the relationship between immune score and prognosis, and then based on this relationship, we developed a clinical nomogram of predicting the survival of LUAD patients. The details were reported as follows:

\section{Methods}

\section{Materials}

The data used in this study were downloaded from The Cancer Genome Atlas database (TCGA). ${ }^{13}$ TCGA is a project funded by the National Cancer Institute (NCI) and National Human Genome Research Institute (NHGRI) and has generated comprehensive and multi-dimensional maps of the key genomic changes in various cancers. TCGA is currently the largest public data set for tumor genome analysis, including more than 200 cancer and clinical information, as well as DNA methylation, gene expression, somatic mutation, etc. ${ }^{14}$ In April 2020, we downloaded the LUAD raw data from the TCGA database (http://www.cbioportal.org).

The immune score data was collected from a public data platform (https://cancergenome.nih.gov/). The immune score was obtained based on the number of lymphocytes in the central area of the tumor and the infiltration junction area. The score suggested the degree of tumor immune cell infiltration. ${ }^{15}$

\section{Data Preprocessing}

The downloaded LUAD case data and the corresponding immune score data were firstly preprocessed to remove cases with incomplete information, and finally the data of $411 \mathrm{LUAD}$ cases were obtained. The $\mathrm{R}$ language software (version 3.6.3) was used to merge the two types of data, the duplicate cases were removed, and finally 407 cases were obtained, for whom there was a consistent one-to-one match between each case and each immune score. Only a very small number of patients were in the advanced stage, and there was no statistical significance. Therefore, the patients in the advanced stage were removed, and finally 390 cases were enrolled for analysis.

The data of cases in this study was processed as follows. The survival status was divided into two categories: 0 meant survival, and 1 meant death. Age was classified into 3 categories: 0 represented " $\leq 50$ years old", 1 represented " $>50$ and 
$\leq 70$ years old", and 2 represented " $>70$ years old". The tumor TNM comprehensive stage was divided into 3 categories: 0 represented "I", 1 represented "II", and 2 represented "III". Immune scores were classified into 3 categories: 0 for "low score ( $\leq 698.1)$ ", 1 for "medium score $(>698.1$ and $\leq 1246.3)$ ", and 2 for "high score $(>1246.3)$ ". There were two categories of gender: 0 for "male", and 1 for "female". Whether or not to undergo radiotherapy was divided into two categories: 0 for "radiotherapy has been carried out", 1 for "radiotherapy has not been carried out". The details of each stage of data preprocessing and the sample size of each stage are shown in Figure 1.

In addition, the data sets were randomly divided into two groups, one was the training set, and the other was the validation set, which was used to do the decision curve analysis (DCA). The DCA is a simple method for evaluating clinical predictive models, diagnostic tests and molecular markers. The abscissa is the threshold probability, which represents the probability that the model predicts the occurrence of an event. The ordinate is the net benefit, which represents When various evaluation methods reach a certain value, the risk probability of patient $\mathrm{i}$ is denoted as $\mathrm{Pi}$. When Pi reaches a certain threshold $(\mathrm{Pt})$, it is defined as positive and some kind of intervention is adopted, which brings the benefits to the patients undergoing treatment, and the harms to the nonpatients undergoing treatment and the patients without treatment. The ordinate is the net benefit of the benefits minus the harms.

\section{Statistical Analysis}

The downloaded data provided the overall survival (OS) time of each patient. OS time was defined as the time from pathological diagnosis to death. ${ }^{16} \mathrm{X}$-tile software (version 3.6.1, Yale University School of Medicine, New Haven, CT, USA) was used to get the cut-off point of the immune score, ${ }^{17}$ and $\mathrm{X}$-tile was used to evaluate the immune score, and the immune score was divided into three groups, as shown in Figure 2.

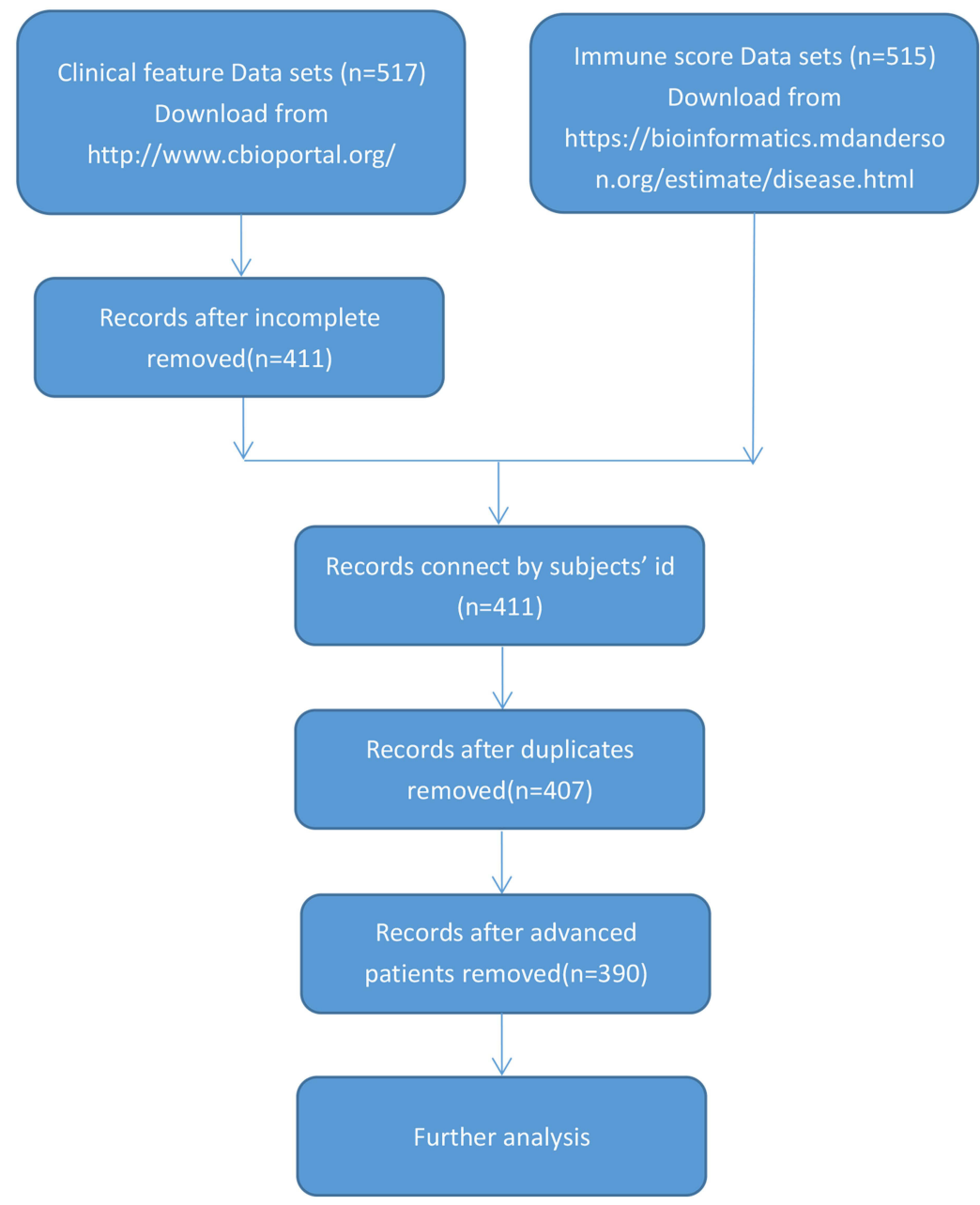

Figure I Research flow chart of the sample collection. 


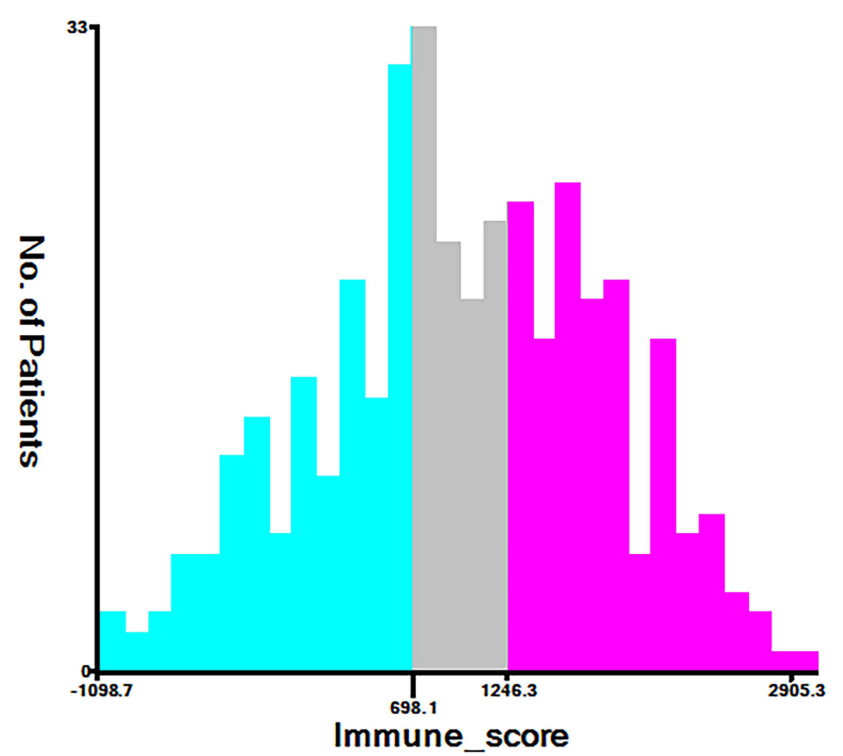

Figure 2 Cutoff values of high, medium, and low immune scores, determined by $\mathrm{X}$-tile software (X-axis representing the immune score, $\mathrm{Y}$-axis representing the number of patients).

Univariate Cox analysis was performed by survival package of R language. All variables with $p<0.05$ in the univariate analysis were included into the multivariate Cox regression model by survival package of $\mathrm{R}$ language. The risk of the immune score combined with the tumor TNM comprehensive stage (I, II, III) was evaluated, and then whether to receive radiotherapy was included in the model. The adjusted hazard ratio (HR) and 95\% confidence intervals (CI) were evaluated. Forest analysis was performed using the survminer package in $\mathrm{R}$ software. On the basis of multivariate Cox regression analysis, "rms and survival" package in R software was used to build a nomogram. A Bootstrap test (1000 replicates) was performed to validate the nomogram. The accuracy of the prognosis was evaluated by the concordance index (c-index) using the survival package in R software. The ROC curves were drawn using survival and timROC packages in $\mathrm{R}$ software. The "rms and survival" package was used to generate a prognostic nomogram for 3- and 5-year OS. The survival curves were drawn by Kaplan-Meier method, and the Log rank test was used to perform the survival analysis.

SPSS 21.0 was used to perform chi-square test on categorical variables. One-way analysis of variance (ANOVA) was used to test the normal distribution and variance homogeneity of the continuous variables. In all statistical tests, $\mathrm{P}<0.05$ was considered statistically significant.

Finally, DCA was performed using $\mathrm{R}$ to evaluate the clinical practical value of the model.

\section{Results}

\section{Patients' Characteristics}

The average age of the enrolled patients was 65.17 years old ( $\mathrm{SD}=10.09$, Range 39-88 years). Twenty-nine patients (7.4\%) were younger than 50 years old, 227 patients (58.2\%) ranged from 51 to 70 years old, and 134 patients (34.4\%) were older than 70 years old. Among the 390 patients, there were $172(44.1 \%)$ males and 218 (55.9\%) females. According to TNM classification, there were 223 (57.2\%) patients in stage I, 105 (26.9\%) patients were in stage II, and $62(15.9 \%)$ patients were in stage III. Patients were divided into groups with high, medium, and low immune scores (Figure 2). A total of 143 (36.7\%) patients with an immune score less than or equal to 698.1 were included in the low score group, $152(39.0 \%)$ patients with an immune score greater than 1246.3 were included in the high score group, and 95 (24.4\%) patients with an immune score greater than 698.1 and less than or equal to 1246.3 were included in the medium score group. Among them, 53 patients (13.6\%) received radiotherapy, and 337 patients (86.4\%) did not receive radiotherapy.

The clinicopathological characteristics of the study subjects in different immune score groups were listed in Table 1. The average age of the high-, medium- and low-score groups were 62.27 years old $(\mathrm{SD}=10.48), 68.05$ years old $(\mathrm{SD}=$ 9.39 ), and 66.24 years old $(\mathrm{SD}=9.42)$, respectively. In the "age $\leq 50$ years old" group, the number of patients with the low immune score was the largest, and so does the "age $>51$ and $\leq 70$ years old" group. Patients who received radiotherapy were evenly distributed in the different immune scores groups. However, among the patients who did not receive radiotherapy, the proportion of patients with the high immune score was the highest. For the female patients, the proportion of patients with the high immune score was the highest, while for the male patients, the proportion of patients with the high immune score was the lowest. In the TNM staging of tumors, stage I and II were more likely to appear in patients with the high immune score.

\section{Univariate and Multivariate Analysis of OS}

The univariate analysis was performed to explore the correlation between the OS and clinicopathological characteristics in LUAD patients. As shown in Table 2 and Figure 3, patients with the low, medium, and high immune scores had significant differences in OS $(\mathrm{P}<0.001)$. In addition, there was a statistically significant difference in 
Table I Correlation Between Clinicopathological Characteristics and Immune Score in 407 Patients with LUAD

\begin{tabular}{|c|c|c|c|c|c|c|}
\hline \multirow[t]{2}{*}{ Characteristics } & \multirow[t]{2}{*}{ Total } & \multicolumn{3}{|c|}{ Immune Scores } & \multirow[t]{2}{*}{$x^{2}$} & \multirow[t]{2}{*}{$P$ value } \\
\hline & & $\leq 698.1$ & 698.1 to I 246.3 & $>1246.3$ & & \\
\hline Sample size & 390 & I 43 (36.7\%) & 95 (24.4\%) & I 52 (39.0\%) & & \\
\hline Age & & & & & 17.635 & 0.001 \\
\hline$\leq 50$ & 29 & $19(13.3)$ & $3(3.2)$ & $7(4.6)$ & & \\
\hline $5 I-70$ & 227 & $89(62.2)$ & $54(56.8)$ & $84(55.3)$ & & \\
\hline$>70$ & 134 & $35(24.5)$ & $38(40.0)$ & $6 I(40.1)$ & & \\
\hline Radiotion & & & & & 0.806 & 0.668 \\
\hline Yes & 53 & $20(14.0)$ & $15(15.8)$ & $18(11.8)$ & & \\
\hline No & 337 & $123(86.0)$ & $80(84.2)$ & 134(88.2) & & \\
\hline Sex & & & & & 8.844 & 0.012 \\
\hline Male & 172 & $77(53.8)$ & $38(40.0)$ & $57(37.5)$ & & \\
\hline Female & 218 & $66(46.2)$ & $57(60.0)$ & $95(62.5)$ & & \\
\hline TNM stage & & & & & 15.494 & 0.004 \\
\hline 1 & 223 & $80(55.9)$ & $45(47.4)$ & $98(64.5)$ & & \\
\hline II & 105 & $4 I(28.7)$ & $24(25.3)$ & $40(26.3)$ & & \\
\hline III & 62 & $22(15.4)$ & $26(27.4)$ & $14(9.2)$ & & \\
\hline
\end{tabular}

Table 2 Univariate Analyses of OS Among LUAD Patients According to Clinic Pathological Characteristics and Immune Scores

\begin{tabular}{|c|c|c|c|c|c|}
\hline \multirow[t]{2}{*}{ Characteristics } & \multirow[t]{2}{*}{ Total } & \multicolumn{4}{|c|}{ Os } \\
\hline & & Survival & Death & HR(95\% CI) & $P$ value \\
\hline \multicolumn{6}{|l|}{ Age } \\
\hline$\leq 50$ & 29 & $20(69.0)$ & $9(31.0)$ & 1.0 & \\
\hline $51-70$ & 227 & I58 (69.6) & $69(30.4)$ & $0.963(0.478-1.938)$ & 0.916 \\
\hline$>70$ & 134 & $76(56.7)$ & $58(43.3)$ & $1.575(0.774-3.205)$ & 0.211 \\
\hline \multicolumn{6}{|l|}{ Sex } \\
\hline Male & 172 & III(64.5) & $6 I(35.5)$ & 1.0 & \\
\hline Female & 218 & $143(65.6)$ & $75(34.4)$ & $0.890(0.634-1.248)$ & 0.499 \\
\hline \multicolumn{6}{|l|}{ Radiation } \\
\hline Yes & 53 & $23(43.4)$ & $30(56.6)$ & 1.0 & \\
\hline No & 337 & $231(68.5)$ & $106(31.5)$ & $0.517(0.344-0.776)$ & 0.0015 \\
\hline \multicolumn{6}{|l|}{ TNM stage } \\
\hline I & 223 & $167(74.9)$ & $56(38.6)$ & 1.0 & \\
\hline II & 105 & $60(57.1)$ & $45(42.9)$ & $2.176(1.462-3.238)$ & $<0.001$ \\
\hline III & 62 & $27(43.5)$ & $35(56.5)$ & $3.100(2.022-4.752)$ & $<0.001$ \\
\hline \multicolumn{6}{|l|}{ Immune scores } \\
\hline$\leq 698.1$ & 143 & $82(57.3)$ & $6 \mathrm{I}(42.7)$ & 1.0 & \\
\hline 698.1 tol 246.3 & 95 & $57(60.0)$ & $38(40.0)$ & $0.875(0.583-1.3 \mid 3)$ & 0.519 \\
\hline$>1246.3$ & 152 & II5 (75.7) & $37(24.3)$ & $0.479(0.318-0.720)$ & $<0.001$ \\
\hline
\end{tabular}

OS of the different TNM stages of tumor $(\mathrm{P}<0.001)$. There was also a difference in OS of patients whether receiving radiotherapy or not $(\mathrm{p}=0.0015)$. However, there were no differences in age and gender $(p>0.05)$.
The results of multivariate Cox proportional hazards regression analysis are shown in Table 3. Compared with patients with low and medium immune scores, the OS of patients with the high immune score was significantly 


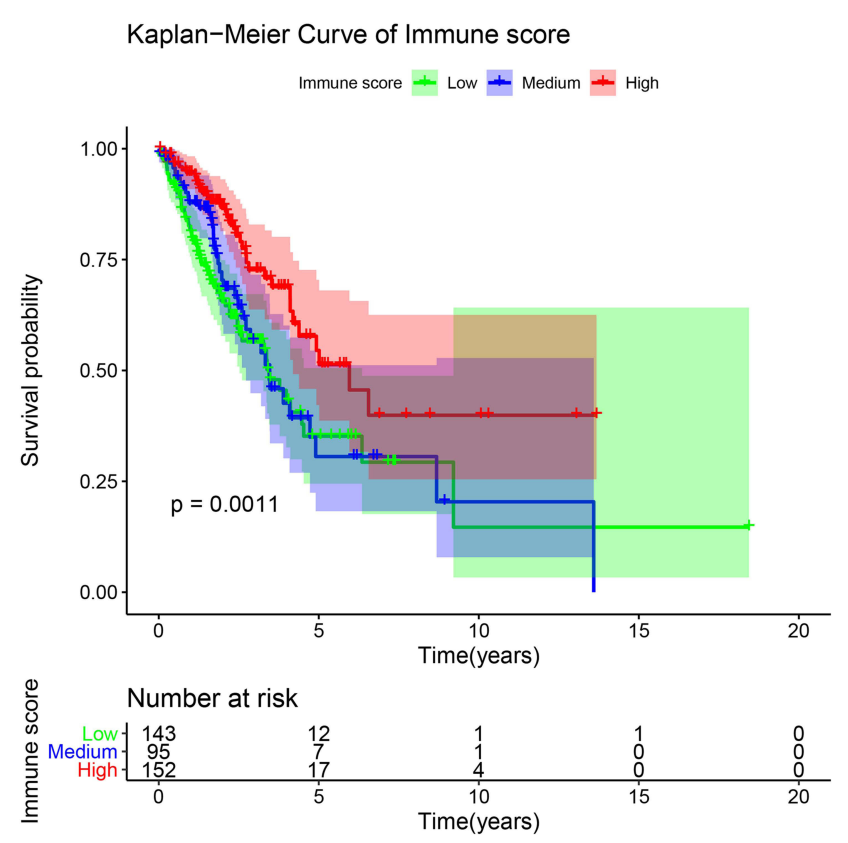

Figure 3 Kaplan-Meier curves showed the associations of immune scores groups with overall survival (OS) of patients with LUAD. Comparison of OS among patients with $\leq 698.1$ immune scores (low group), patients with immune scores between 698.1 and 1246.3 (medium group), and patients with $>1246.3$ immune scores (high group).

improved (HR and 95\% CI: 0.489 [0.324-0.737]). For the tumor staging, the OS of patients in the stage II and III was significantly worse than those in the stage I, and HR and 95\% CI for stages II and III were 2.167 [1.450-3.238] and 2.588 [1.636-4.094], respectively. Patients who did not receive radiotherapy had worse OS than those who received radiotherapy, with $\mathrm{HR}$ and $95 \%$ CI $(0.640$ [0.414-0.989]). Therefore, whether to receive radiotherapy or not, the TNM stage and the immune score were included in the multivariate model.

\section{Prognostic Nomogram of OS}

Cox regression model was used to evaluate the associations between potential prognostic factors and OS by univariate and multivariate analysis. The potential prognostic factors included the immune score, tumor staging and whether to receive radiotherapy. According to the results of the multivariate Cox regression analysis, the forest curve was drawn. The forest plot showed the relationship between the potential prognostic factors and OS (Figure 4).

The prognostic nomogram of OS, which integrated all significant independent factors obtained from the multivariate analysis, is shown in Figure 5 . The $\mathrm{C}$-index for predicting OS was 0.691 (95\% CI, 0.646-0.736). Based on the above analysis, a receiver-operator-characteristics (ROC) curve was drawn, and the area under the curve (AUC) was calculated. The AUC values of 3- and 5-year OS were 0.698 and 0.684 , respectively (Figure 6). The reliability of the prediction model was verified by the $\mathrm{C}$ index and ROC curve.

The calibration curves of 3- and 5-year OS showed a good consistency in the probability between the actual observation and the nomogram prediction (Figure 7A and $\mathrm{B})$.

\section{Decision Curve Analysis}

In the DCA chart, there are four curves. The black None line represented that all patients did not have LUAD and did not receive any treatment, and the net benefit (Net Benefit) was 0 . The gray All line represented that all patients had LUAD and received the treatment, and the net benefit was a backslash with a negative slope.

Table 3 Multivariate Analyses of OS Among LUAD Patients According to Immune Scores and Clinic Pathological Characteristics

\begin{tabular}{|c|c|c|c|c|c|}
\hline \multirow[t]{2}{*}{ Characteristics } & \multirow[t]{2}{*}{ Total } & \multicolumn{4}{|c|}{ os } \\
\hline & & Survival & Death & HR (95\% Cl) & $P$ value \\
\hline \multicolumn{6}{|l|}{ Radiation } \\
\hline Yes & 53 & $23(43.4)$ & $30(56.6)$ & 1.0 & \\
\hline No & 337 & $231(68.5)$ & $106(31.5)$ & $0.640(0.4 \mid 4,0.989)$ & 0.044 \\
\hline \multicolumn{6}{|l|}{ TNM stage } \\
\hline I & 223 & I67 (74.9) & $56(38.6)$ & I & \\
\hline II & 105 & $60(57.1)$ & $45(42.9)$ & $2.167(1.450,3.238)$ & $<0.001$ \\
\hline III & 62 & $27(43.5)$ & $35(56.5)$ & $2.588(1.636,4.094)$ & $<0.001$ \\
\hline \multicolumn{6}{|l|}{ Immune scores } \\
\hline$\leq 683.7$ & 143 & $82(57.3)$ & $6 \mathrm{I}(42.7)$ & 1 & \\
\hline 683.7 tol 246.3 & 95 & $57(60.0)$ & $38(40.0)$ & $0.828(0.548, I .25 \mathrm{I})$ & 0.371 \\
\hline $\mid 246.3>$ & 152 & II 5 (75.7) & $37(24.3)$ & $0.489(0.324,0.737)$ & $<0.001$ \\
\hline
\end{tabular}




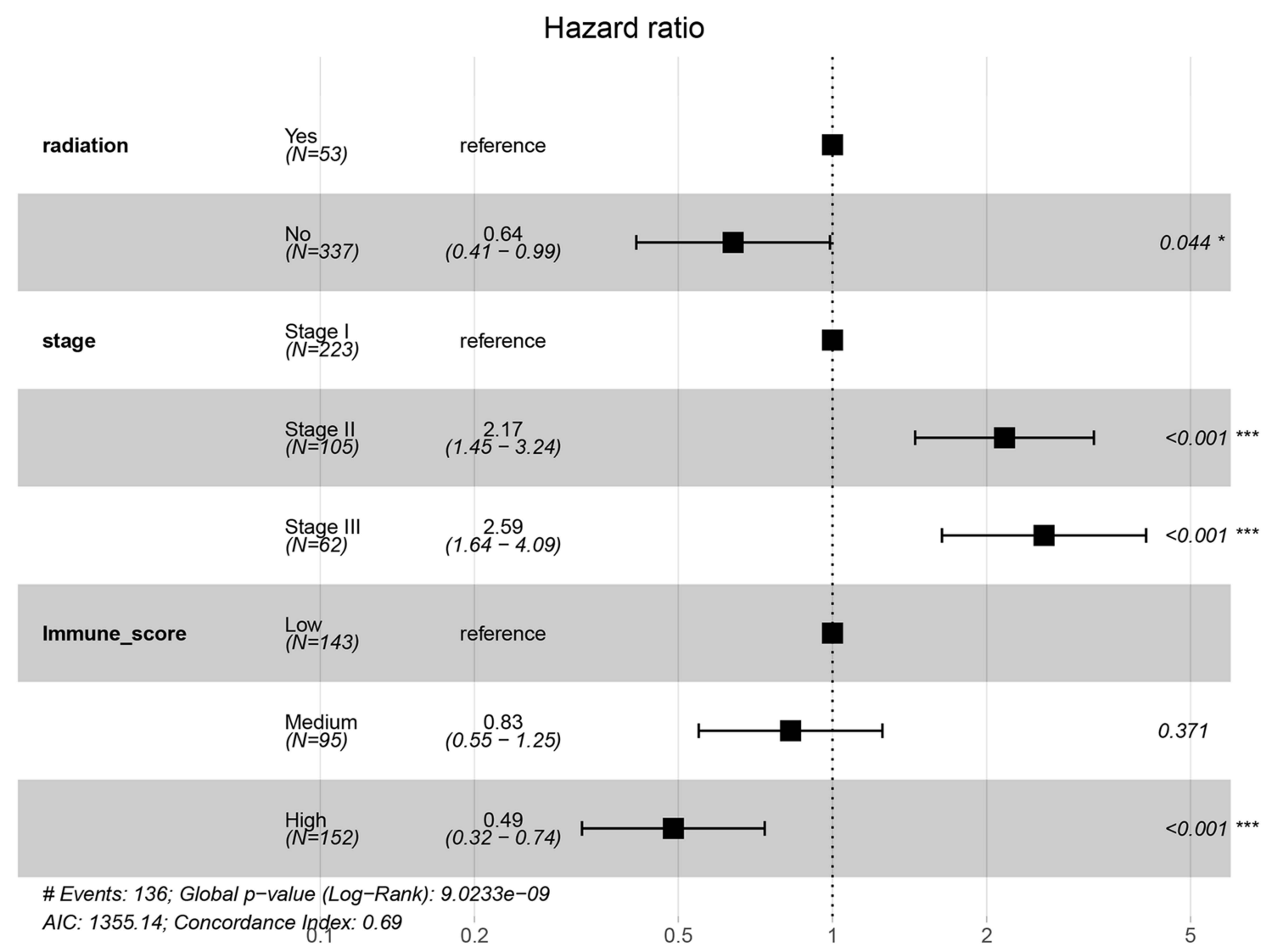

Figure 4 Forest plot of overall survival (OS). $* P<0.05, * * * P<0.001$.

Points

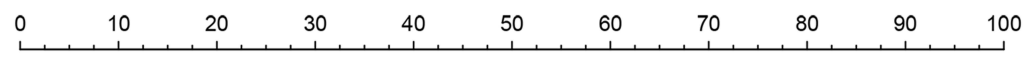

radiation

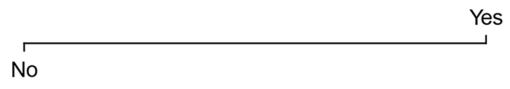

stage

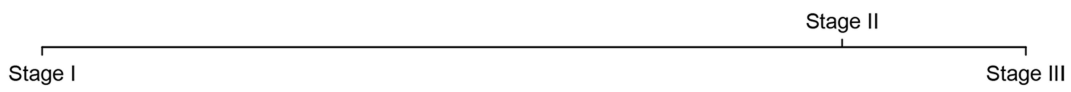

Immune_score

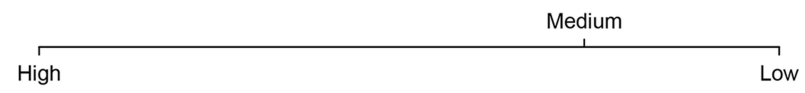

Total Points

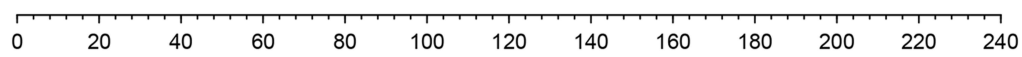

3-Year Survival

\begin{tabular}{lllllll}
\hline 0.8 & 0.7 & 0.6 & 0.5 & 0.4 & 0.3 & 0.2
\end{tabular}

5-Year survival

$\begin{array}{lllllll} & . & 1 & 1 & & \\ & 0.6 & 0.5 & 0.4 & 0.3 & 0.2 & 0.1\end{array}$

Figure 5 Nomograms for predicting survival of LUAD. 


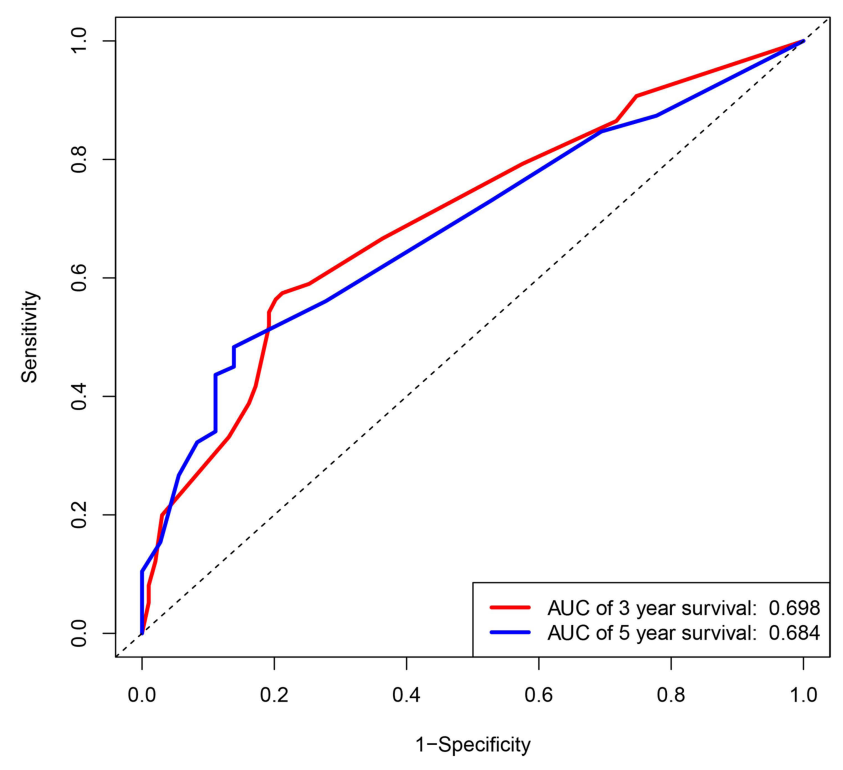

Figure 6 The ROC curve of overall survival (OS) of 3 and 5 years for the LUAD patients.

In the group of the training set, the blue Immune_score curve was above the black None line and the gray All line, when the high-risk threshold value ranged from 0.20 to 0.52 , suggesting the model had clinical practical value, and the red Nomogram curve was above the black None line and the gray All line, when the high-risk threshold value ranged from 0.20 to 0.71 , suggesting the model had clinical practical value (Figure 8A).

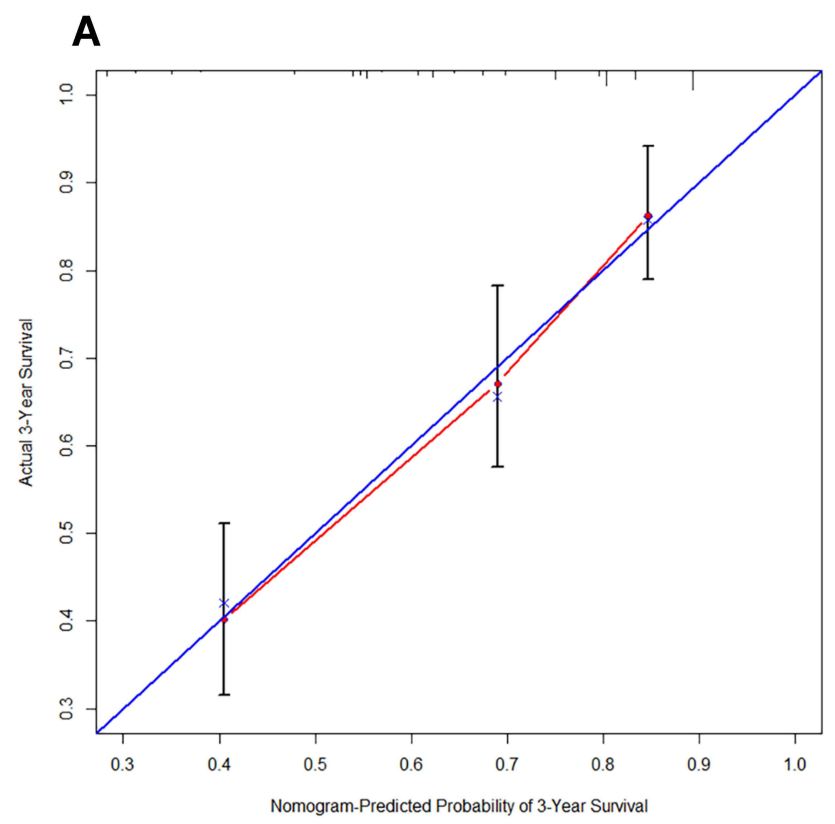

In the group of the validation set, the blue Immune_score curve was above the black None line and the gray All line, when the high-risk threshold value ranged from 0.24 to 0.36 , suggesting the model had clinical practical value, and the red Nomogram curve was above the black None line and the gray All line, when the high-risk threshold value ranged from 0.12 to 0.60 , suggesting the model had clinical practical value (Figure 8B).

The DCA of the two data sets both confirmed that the model constructed in this study had clinical practical value.

\section{Discussion}

In this study, publicly available data from the TCGA repositories was used to identify the immune score associated with OS of LUAD patients in the stages I, II, and III. After adjusting for possible confounding factors, we found that the high immune score was significantly associated with OS of LUAD patients. At the same time, a nomogram of predicting the survival time of LUAD patients was established.

The important contribution of immune cells to LUAD has been widely accepted, ${ }^{18}$ and immune genes were considered as biomarkers of immune response in immunotherapy. ${ }^{19}$ Early studies also showed that some immune genes were significantly related to the prognosis

\section{B}

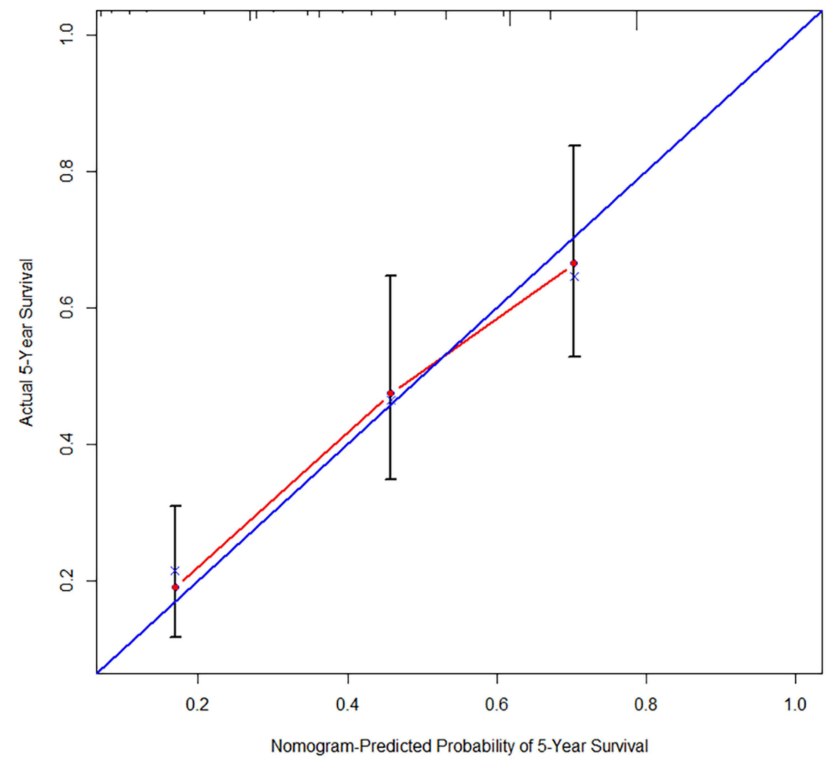

Figure 7 Calibration curve of 3-year (A) and 5-year (B) overall survival (OS) for the LUAD patients. Nomogram-predicted probability of OS was expressed by the x-axis; actual OS was expressed by the $y$-axis. 

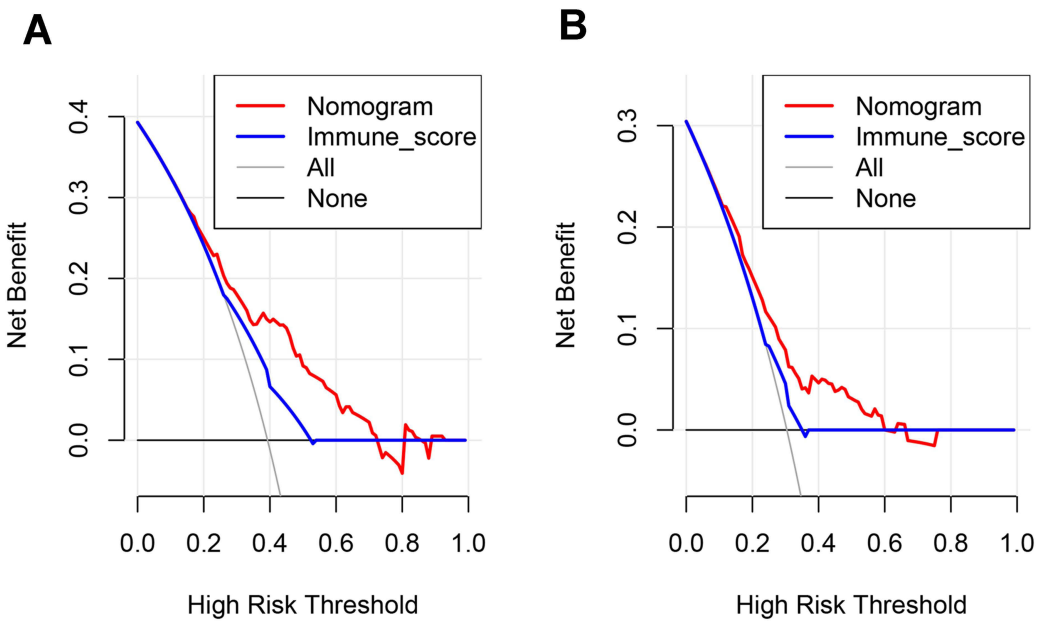

Figure 8 Decision curve analysis of the training set group $(\mathbf{A})$ and the validation set group (B).

of LUAD. $^{20}$ In addition, some studies suggested that immune gene expression should be included in the current multi-gene test to improve the prognosis of patients with LUAD. ${ }^{21,22}$ However, these findings have not been used in clinical studies to predict the probability of OS of LUAD. In addition, few studies established a nomogram using the immune score. In this study, the clinicopathological information and the immune scores of LUAD patients from the TCGA were used to explore their association with prognosis. Furthermore, a nomogram was established to make the prognostic assessment of LUAD patients easier.

In this study, after adjusting for several possible confounding factors, we found the higher the immune score was, the higher the OS of LUAD patients was. Similar results were observed in the study of Pagès et al. ${ }^{23}$ The possible reason might be that the high immune score meant that the immune system and immune function were enhanced, which could improve the anti-tumor immunity of the tumor microenvironment, thereby controlling and eliminating the tumor. ${ }^{24}$ In addition, related studies found that genes related to immune cell activation significantly increased in relatively long-lived patients with cancer. ${ }^{25}$ Moreover, some important genes, such as CD302, etc., were used to calculate immune characteristics and play important roles in immune function. ${ }^{26}$ Furthermore, a study on $\mathrm{T}$ cell-related markers indicated that LUAD patients with higher expression of $\mathrm{CD} 3$ + and $\mathrm{CD} 8+$ receiving relevant neoadjuvant chemotherapy had a good prognosis. ${ }^{27}$ Therefore, the immune score could not only be used as a biomarker for the prognosis of LUAD patients but also has a potential clinical value in the selection of treatment strategies.
In this study, we found that whether to receive radiotherapy was an important independent prognostic factor for patients with $\operatorname{LUAD}(\mathrm{p}=0.0015)$. Patients who received radiotherapy had better OS, with HR: 0.517 and 95\% CI: $0.344-0.776$. With the improvement of the awareness of early cancer screening among citizens and the level of medical and health care, the early detection rate of cancer increased significantly, which was similar to our study in which more than $50 \%$ of the patients enrolled were in the stage I. However, our results were different from previous studies. The previous studies suggested that most of the LUAD patents were in the advanced stage (III/IV) at the time of initial diagnosis, and the best opportunity for surgery had been missed. ${ }^{28,29}$ Early detection greatly improves the success rate of the patients' treatment and prolongs the patients' survival time. For LUAD patients in the early stage, surgery is the main treatment supplemented by adjuvant radiotherapy and chemotherapy. However, some studies reported that long-term chemical/radiotherapy had the possibility of carcinogenesis. ${ }^{30}$ Postoperative radiotherapy and chemotherapy can reduce the patient's own immunity and weaken the defense against the tumor, which is conducive to the tumor regeneration. For LUAD patients with high immune scores before and after surgery, immunotherapy or combined immunotherapy based on precise gene sequencing can reduce the harm of radiotherapy and chemotherapy. ${ }^{31}$ Studies reported that postoperative immunotherapy could generally reduce the local recurrence rate by about $30 \%$, significantly reduce distant metastases, and increase the 5-year survival rate by 
about $20 \% .{ }^{32}$ Even if the tumor recurred, the recurrence time could be obviously delayed. ${ }^{32}$ It was confirmed that for treating the resectable non-small cell lung cancer, neoadjuvant immunotherapy was highly safe and did not affect surgery, with $45 \%$ of the pathologically significant remission rate and $73 \%$ of the 18 -month recurrence-free survival rate. ${ }^{33}$ Studies have also shown that for the earlystage non-small cell cancer, immune drugs can block a variety of molecular markers to kill cancer cells with fewer side effects. ${ }^{34}$ Therefore, in addition to the traditional lung cancer treatment, immunotherapy can improve the survival rate of patients. ${ }^{34}$

The LUAD prognostic model was constructed based on the immune cell infiltration score and clinicopathological characteristics, clarifying the relationship between immune cell infiltration and the occurrence and development of LUAD. The prognostic model we established could effectively predict the 3- and 5-year survival rates of LUAD patients, which suggested the roles of different immune scores in the development of LUAD. These findings might provide new ideas for the treatment and prognosis of LUAD from the perspective of immune cell infiltration.

However, there are still some limitations in our study. There were relatively few gene expression data sets used to calculate immune scores. The LUAD patients in the stage IV were not enrolled in this study. We only made the statistical analysis of the treatment of whether to receive the radiotherapy or not and did not analyze the other treatment methods for LUAD patients. No external data verification was performed. In addition, the nomogram is a little simple and has a limited effect in the accurate prediction of the survival of patients, and thus in the future, the more comprehensive prognostic model will be established to improve the accuracy of predicting the survival of patients. For us, there is still a lot of work to do in the future. In addition to clinical pathological factors, further efforts will be made to collect case data related to immune gene expression, thereby updating and developing our model.

\section{Conclusions}

This study found that for LUAD patients in the stages I, II, and III, the high immune score was significantly associated with better OS. In addition, the established nomogram could effectively predict the prognosis of LUAD. This practical prognostic model can easily evaluate the OS of patients and identify the patients who need active treatment.

\section{Abbreviations}

NSCLC, non-small cell lung cancer; OS, overall survival; LUAD, lung adenocarcinoma; HR, hazard ratios; CI, confidence intervals; c-index, concordance index; EGFR, epidermal growth factor receptor; TME, the tumor microenvironment; $\mathrm{PD}-1$, programmed cell death protein 1; PD-L1, its ligand (PD-L1); TCGA, The Cancer Genome Atlas; NCI, National Cancer Institute; NHGRI, National Human Genome Research Institute; ROC, receiveroperator-characteristics; AUC, area under the curve.

\section{Data Sharing Statement}

The datasets used and/or analysed during the current study are available from the corresponding author on reasonable request.

\section{Ethics Approval and Consent to Participate}

Not applicable, because TCGA belongs to public databases, the patients involved in the database have obtained ethical approval, users can download relevant data for free for research and publish relevant articles, and our study is based on open-source data, and the Affiliated Hospital (Clinical College) of Xiangnan University do not require research using publicly available data to be submitted for review to their ethics committee, so there are no ethical issues and other conflicts of interest.

\section{Acknowledgments}

Thanks for the support from the funders and TCGA database.

\section{Funding}

This study was supported by:1. Science and Technology Funding Project of Hunan Province China (No.2017SK4010)2. Key Laboratory of Tumor Precision Medicine, Hunan colleges and Universities Project (2019379)3. Science and Technology Project of Chenzhou city (No. CZKJ2016031)The funding bodies played no role in the design of the study and collection, analysis, and interpretation of data and in writing the manuscript, but paid our wages, and thus we can concentrate on the studies to get more achievements.

\section{Disclosure}

The authors declare that they have no conflicts to disclose. 


\section{References}

1. He Y, Liang D, Li D, et al. Incid ence and morta lity of laryngeal cancer in China, 2015. Chin J Cancer Res. 2020;32(1):10-17.

2. Fitzmaurice C, Abate D, Abbasi N; Global Burden of Disease Cancer Collaboration. Global, regional, and national cancer incidence, mortality, years of life lost, years lived with disability, and disability-adjusted life-years for 29 cancer groups, 1990 to 2017: a systematic analysis for the global burden of disease study. JAMA Oncol. 2019;5(12):1749-1768. doi:10.1001/jamaoncol.2019.2996

3. Cortinovis D, Abbate M, Bidoli P, Capici S, Canova S. Targeted therapies and immunotherapy in non-small-cell lung cancer. Ecancermedicalscience. 2016;10:648. doi:10.3332/ecancer.2016.648

4. Wang D, Gao Y, Zhang Y, Wang L, Chen G. Glypican-3 promotes cell proliferation and tumorigenesis through up-regulation of $\beta$ catenin expression in lung squamous cell carcinoma. Biosci Rep. 2019;39(6):BSR20181147. doi:10.1042/BSR20181147

5. Thunnissen E, Boers E, Heideman DA, et al. Correlation of immunohistochemical staining p63 and TTF-1 with EGFR and K-ras mutational spectrum and diagnostic reproducibility in non small cell lung carcinoma. Virchows Arch. 2012;461:629-638. doi:10.1007/ s00428-012-1324-x

6. Sugiyama E, Togashi Y, Takeuchi Y, et al.Blockade of EGFR improves responsiveness to PD-1 blockade in EGFR -mutated nonsmall cell lung cancer. Immunology. 2020;5(43):eaav3937.

7. Domagala-Kulawik J. The role of the immune system in non-small cell lung carcinoma and potential for therapeutic intervention. Transl Lung Cancer Res. 2015;4(2):177-190. doi:10.3978/j.issn.22186751.2015.01.11

8. Garon EB, Hellmann MD, Rizvi NA, et al. Five-year overall survival for patients with advanced non-small-cell lung cancer treated with pembrolizumab: results from the phase I KEYNOTE-001 study. JClin Oncol. 2019;37(28):2518-2527. doi:10.1200/JCO.19.00934

9. Reck M, Rodriguez-Abreu D, Robinson AG, et al. Pembrolizumab versus chemotherapy for PD-L1-positive non-small-cell lung cancer. N Engl J Med. 2016;375:1823-1833. doi:10.1056/NEJMoa1606774

10. Ren S, Tian Q, Amar N. The immune checkpoint, HVEM may contribute to immune escape in non-small cell lung cancer lacking PD-L1 expression. Lung Cancer. 2018;125:115-120. doi:10.1016/j. lungcan.2018.09.004

11. Jiang Y, Zhang Q, Hu Y, et al. ImmunoScore signature: a prognostic and predictive tool in gastric cancer. Ann Surg. 2016. doi:10.1097/ SLA.0000000000002116

12. Wang J, Li Y, Fu W, et al. Prognostic nomogram based on immune scores for breast cancer patients. Cancer Med. 2019;8:5214-5222. doi:10.1002/cam4.2428

13. Weinstein JN, Collisson EA, Mills GB, et al. The cancer genome atlas pan-cancer analysis project. Nat Genet. 2013;45:1113-1120. doi:10.1038/ng.2764

14. Oliveira-Mateos C, Sánchez-Castillo A, Soler M, et al. The transcribed pseudogene RPSAP52 enhances the oncofetal HMGA2-IGF2BP2-RAS axis through LIN28B-dependent and independent let-7 inhibition. Nat Commun. 2019;10(1):3979. doi:10.1038/ s41467-019-11910-6

15. Miyashita M, Sasano H, Tamaki K, et al. Prognostic significance of tumor-infiltrating $\mathrm{CD} 8+$ and FOXP3+ lymphocytes in residual tumors and alterations in these parameters after neoadjuvant chemotherapy in triple-negative breast cancer: a retrospective multicenter study. Breast Cancer Res. 2015;17(1):124. doi:10.1186/s13058-015-0632-x

16. Chen Y, Li W, Tang W, Yang X, Zhong W. [Observation - an favorable option for thoracic dissemination patients with lung adenocarcinoma or squamous carcinoma]. Zhongguo Fei Ai Za Zhi. 2018;21(4):303-309. Chinese. doi:10.3779/j.issn.1009-3419.2018.04.14

17. Available from: https://medicine.yale.edu/lab/rimm/research/soft ware/. Accessed November 19, 2021.
18. Sugiyama E, Togashi Y, Takeuchi Y, et al. Blockade of EGFR improves responsiveness to PD-1 blockade in EGFR-mutated nonsmall cell lung cancer. Sci Immunol. 2020;5. doi:10.1126/sciimmunol.aav3937

19. Anagnostou V, Niknafs N, Marrone K, et al. Multimodal genomic features predict outcome of immune checkpoint blockade in non-small-cell lung cancer. Nature Cancer. 2020;1:99-111. doi:10.1038/s43018-019-0008-8

20. Yu Y, Zeng D, Ou Q, et al. Association of survival and immunerelated biomarkers with immunotherapy in patients with non-small cell lung cancer. JAMA Netw Open. 2019;2(7):e196879. doi:10.1001/ jamanetworkopen.2019.6879

21. Rizvi NA, Hellmann MD, Snyder A, et al. Mutational landscape determines sensitivity to PD-1 blockade in non-small cell lung cancer. Science. 2015;348(6230):124-128. doi:10.1126/science. aaa1348

22. Dai F, Liu L, Che G, et al. The number and microlocalization of tumor-associated immune cells are associated with patient's survival time in non-small cell lung cancer. BMC Cancer. 2010;10(1):220. doi:10.1186/1471-2407-10-220

23. Pagès F, Kirilovsky A, Mlecnik B, et al. In situ cytotoxic and memoryT cells predict outcome in patients with early-stage colorectal cancer.J. Clin Oncol. 2009;27(35):5944-5951. doi:10.1200/ JCO.2008.19.6147

24. Chen DS, Mellman I. Elements of cancer immunity and the cancer-immune set point. Nature. 2017;541:321-330. doi:10.1038/ nature 21349

25. Constant SL, Bottomly K. Induction of Th1 and Th2 CD4+ T cell responses: the alternative approaches. Annu Rev Immunol. 1997;15 (15):297-322. doi:10.1146/annurev.immunol.15.1.297

26. Yoshihara K, Shahmoradgoli M, Martínez E, et al. Inferring tumour purity and stromal and immune cell admixture from expression data. Nat Commun. 2013;4:2612. doi:10.1038/ ncomms 3612

27. Yan X, Jiao S. Roles of tumor-infiltrating lymphocytes in non-small cell lung cancer recurrence and metastasis: a meta analysis. Acta Academiae Medicinae Sinicae. 2015;37(4):406-414. doi:10.3881/j. issn.1000-503X.2015.04.007

28. Fan H, Shao ZY, Xiao YY, et al. Survival analysis of patients with lung cancer in Shanghai. Chin J Tuberc Respir Dis. 1993;16 (1):36-38.

29. Gutierrez A, Suh R, Abtin F, Genshaft S, Brown K. Lung cancer screening. Semin Intervent Radiol. 2013;30(2):114-120. doi:10.1055/ s-0033-1342951

30. He P, Gu X, Guan YB, et al. Clinicopathologic analysis of 37 cases of synchronous multiple primary lung cancer. Zhonghua Zhong Liu Fang Zhi Za Zhi. 2013;20(5):357-360.

31. Li N, Ying J, Tao X, et al. P1.18-06 efficacy and safety of neoadjuvant PD-1 blockade with sintilimab in resectable non-small cell lung cancer. $J$ Thorac Oncol. 2019;14(10):S627-S628. doi:10.1016/j. jtho.2019.08.1322

32. Herbst RS, Garon EB, Kim D-W, et al. Long-term survival in patients (pts) with advanced NSCLC in the KEYNOTE-010 study overall and in pts who completed two years of pembrolizumab (pembro). Ann Oncol. 2018;29(Supl.8):viii749-viii749. doi:10.1093/annonc/ mdy 424.075

33. Forde PM, Chaft JE, Smith KN, et al. Neoadjuvant PD-1 blockade in resectable lung cancer. $N$ Engl $J$ Med. 2018;378:1976-1986. doi:10.1056/NEJMoa1716078

34. Forde PM, Brahmer JR, Kelly RJ. New strategies in lung cancer: epigenetic therapy for non-small cell lung cancer. Clin Cancer Res. 2014;20(9):2244-2248. doi:10.1158/1078-0432.ccr-13-2088

35. Travis WD, Brambilla E, Riely GJ. New pathologic classification of lung cancer: relevance for clinical practice and clinical trials. J Clin Oncol. 2013;31:992-1001. 


\section{Publish your work in this journal}

The International Journal of General Medicine is an international, peer-reviewed open-access journal that focuses on general and internal medicine, pathogenesis, epidemiology, diagnosis, monitoring and treatment protocols. The journal is characterized by the rapid reporting of reviews, original research and clinical studies across all disease areas. The manuscript management system is completely online and includes a very quick and fair peer-review system, which is all easy to use. Visit http://www.dovepress.com/ testimonials.php to read real quotes from published authors. 